Assessment, Development and Evaluation (GRADE). Modalities included ultrasound, magnetic resonance imaging (MRI), radiographs, positron emission tomography (PET), bone scintigraphy and computerised tomography (CT) Results: 6569 abstracts were screened and 56 papers were included. 50 studies did not adjust for covariates. The majority of studies showed conflicting findings. There was no significant association between most imaging features (rotator cuff tears, tendinopathies, subacromial bursal pathologies, osteoarthritis, calcification, acromial pathologies and adhesive capsulitis) and symptoms amongst high quality, cross-sectional studies. There was low-quality evidence suggesting that enhancement of the joint capsule on MRI and increased uptake of the rotator cuff interval, anterior joint capsule or axillary recess on PET was associated with symptoms in adhesive capsulitis. Based on high-quality, unadjusted longitudinal studies, enlarging rotator cuff tears was associated with an increased incidence of pain. 20 out of 56 studies evaluated more than one pathology, but only one study examined the association of symptoms with a combination of pathologies.

Conclusions: There were conflicting results on the association of imaging features with shoulder symptoms and its persistence and the existing evidence was very low in quality. There may be some imaging features associated with adhesive capsulitis symptoms and increasing RC tear may be associated with incident shoulder symptoms. Further high quality studies are required to understand the relationship between imaging and symptoms.

Disclosure of Interest: None declared

DOI: 10.1136/annrheumdis-2018-eular.2549

\section{AB1188 NAREDO AND BACKHAUS ULTRASOUND SCORES IN TUNISIAN RHEUMATOID ARTHRITIS PATIENTS}

S. ZROUR ${ }^{1}$, H. Mouanaa ${ }^{1}$, A. Arfa ${ }^{1}$, H. Hachfi ${ }^{2}$, M. Jguirim $^{1}$, I. bejia ${ }^{1}$, T. Mongi ${ }^{1}$ M. Youness ${ }^{2}$, N. bergaoui ${ }^{1} .{ }^{1}$ RHEUMATOLOGY DEPARTEMENT OF MONASTIR UNIVERSITY HOSPITAL, monastir, ${ }^{2}$ RHEUMATOLOGY DEPARTEMENT OF MAHDIA UNIVERSITY HOSPITAL, Mahdia, Tunisia

Background: Ultrasound becomes increasingly important in the diagnosis and management of rheumatoid arthritis (RA).

Objectives: Our purposes are to evaluate Naredo (NS) and Backhaus (BS) ultrasound scores of synovitis, performed in daily practice in RA and to study their correlations with clinical assessment and SHARP radiological score modified by Vander Heijde (SS).

Methods: This is a cross-sectional study, conducted at the Rheumatology Department of Monastir Teaching Hospital, Tunisia, in 69 consecutive patients with RA. For each patient, clinical and biological evaluation (DAS 28 score), radiological assessment (SS) and ultrasound evaluation (by TOSHIBA machine) to determine NS and BS, were performed.

Results: The mean age of our patients was $52.01 \pm 10.1$ years. ${ }^{27-78}$ The women accounted for $89 \%$ of patients. The average of disease duration was $121 \pm 86$ months [1-333]. The mean tender joint count (TJC) was 5.9 \pm 5.6 [0-27]. The most frequently hands tender joints were the 5th MCP right, 3rd MCP right and the 1st MCP left and right. The mean swollen joint count (SJC) was 3 [0-22]. The most frequently swollen joints at both hands were the wrists and the 2nd MCP. The overall mean SS score was $105 \pm 59$ [17-272], the overall mean score for joint erosion was $52 \pm 38[0-166]$ and narrowing score was $53 \pm 26$. $^{3-136}$

The mean NS was $17 \pm 15[0-64]$ and BS was $21 \pm 10 .^{2-44}$ The mean duration of ultrasound assessment for calculating NS and BS was $21 \pm 7$ min and $17 \pm 5 \mathrm{~min}$, respectively. NS was significantly associated with SJC $(p<0.000)$ and DAS 28 $(p<0.01)$ but was not significantly associated with SS nor with its components. The BS was significantly associated with TJC $(p<0.000)$, SJC $(p<0.04)$ and DAS $28(p<0.02)$. It was not significantly associated with $S S$. The number of erosions found by the BS was significantly associated with the erosion score of both hands and overall erosion score found by the SS $(p<0.001)$. Ultrasound shows superiority in detecting erosions than standard radiographs: the number of erosions found by the BS was superior in $91 \%$ of cases to the number of erosions found by SS in the same joints.

Conclusions: NS and BS are significantly associated with RA disease activity Ultrasound detects better osteoarticular erosions than radiological assessment. $\mathrm{BS}$, which needs on average $17 \mathrm{~min}$, can be used in daily practice.

\section{REFERENCE:}

1 Contant, Elise, Paul Ornetti. "Ultrasound Scores: Interest in rheumatologic prac tice(example of rheumatoid arthritis and spondyloarthritis)". Journal of Rheumatism Monographs 2005 September;82(4):217-24.

Disclosure of Interest: None declared

DOI: 10.1136/annrheumdis-2018-eular.5763

\section{$\mathrm{AB} 1189$}

ROLE OF DIFFUSION WEIGHTED IMAGING IN DIABETIC FOOT MAGNETIC RESONANCE IMAGING

H. Zaghouani Ben Alaya ${ }^{1}$, F. Ferhi ${ }^{2} .{ }^{1}$ departement of radiology; ${ }^{2}$ anesthesia and intensive care, university hospital farhat hached susa, susa, Tunisia

Background: Differentiation of acute diabetic osteoarthropathy from acute osteomyelitis is one of the most challenging issues in the evaluation of diabetes related foot complications. Early differentiation between these conditions is crucial, as early management of acute diabetic osteoarthropathy could prevent permanen deformity and resultant morbidity and initiation of appropriate treatment for osteomyelitis can reduce the risk of amputations. Acute diabetic osteoarthropathy may mimic osteomyelitis clinically and at different imaging modalities. The current imaging modalities used for assessment for diabetic foot have several limitations Objectives: evaluate the role of diffusion weighted echoplanar MR imaging in differentiation of diabetic osteoarthopathy from osteomyelitis of diabetic foot.

Methods: Prospective study was conducted on 37 consecutive patients with diabetic foot. They underwent diffusion weighted MR imaging of the foot using a single shot echo planar imaging with a b-factor of 0,500 and $1000 \mathrm{sec} / \mathrm{mm} 2$. The scanning parameters were: $\mathrm{TR}=10000 \mathrm{~ms}, \mathrm{TE}=108 \mathrm{~ms}, \mathrm{NEX}=8-16$, band width $=125 \mathrm{kHz}$, slice thickness $=4 \mathrm{~mm}$. Apparent diffusion coefficient (ADC) map was reconstructed. The ADC value was calculated and correlated with surgical findings or biopsy. Statistical analysis was done.

Results: The mean ADC value of diabetic osteoarthropathy was $0.97 \pm 0.13 \times 10$ $3 \mathrm{~mm} 2 / \mathrm{sec}$ and of osteomyelitis was $0.121 \pm 0.12 \times 10-3 \mathrm{~mm}^{2} / \mathrm{sec}$. There was statistically difference in mean ADC values between diabetic osteoarthtopathy and osteomyelitis $(p<0.01)$. When apparent diffusion coefficient value of $0.77 \times$ 10-3 mm2/sec was used as a threshold value for differentiating of diabetic osteoartrhopathy from osteomyelitis, the best result was obtained with an accuracy of $90 \%$, sensitivity $92 \%$, specificity $89 \%$, positive predictive value $88 \%$ and negative predictive value of $86 \%$.

Conclusions: we concluded that apparent diffusion coefficient value is a new non-invasive imaging parameter that can be used for differentiation of diabetic osteoarthropathy from osteomyelitis. Application Diffusion weighted MR imaging can be added to routine MR imaging of diabetic foot.

Disclosure of Interest: None declared

DOI: 10.1136/annrheumdis-2018-eular.5409

\section{AB1190 ULTRASONOGRAPHY IN SPAINSH RHEUMATOLOGY: A CROSS SECTIONAL SURVEY}

J. Uson ${ }^{1}$, E. Naredo ${ }^{2}$, E. de Miguel ${ }^{3}$, J.L. Andreu ${ }^{4} .{ }^{1}$ Rheumatology, Hospital Universitario Móstoles; ${ }^{2}$ Rheumatology, Hospital Fundación Jiménez Díaz; ${ }^{3}$ Rheumatology, Hospital Universitario La Paz; ${ }^{4}$ Rheumatology, Hospital Universitario Puerta de Hierrode Majahahonda, Madrid, Spain

Background: Ultrasonography (US) is the rheumatologísts' best tool that must be taught and learnt correctly. The ultrasound school of the Spanish Society of Rheumatology has trained many rheumatologists for more than twenty years. Approximately $75 \%$ of the rheumatology departments in Spain use US. However, we lack current views of features related with competency, teaching rheumatology residents, US usage and implementation.

Methods: A cross sectional survey was performed using an online standardised questionnaire sent to all members of the SSR in January 2017. The questionnaire was developed by the four authors, corrected for size, style, syntaxes and was piloted on four different aged rheumatologists. Six aspects were studied; general data, US training, rheumatology residents training in US and main uses and applications of US. The questionnaire used either closed or Likert scale answers and took about $20 \mathrm{~min}$ to answer. Herein we describe the main results on usage and applications of US.

Results: 113 responses: $60 \%$ women, $45 \pm 12$ years old, $73 \%$ working in public university hospital with one or more US machines. US is usually used at the time of clinical consultation or at appointment for US. $43 \%$ have a specific agenda for US diagnosis and monitoring inflammatory arthritis patients. The 3 principals for using US are: as a problem solving tool in the clinical context; as a diagnostic tool in inflammatory arthritis and; as a tool to guide injections. The table shows the percentage of the responses always and almost always for 10 clinical contexts. As diagnostic tool in rheumatoid arthritis (RA), 66\% use US to confirm arthritis when clinical exam is unclear and $33 \%$ in patients with inflammatory arthralgia plus high ESR, CRP, RF or anti-CCP. $36 \%$ monitor disease activity using a reduced joint assessment; one target joint plus 2,3 MCP and 2,3, 5 MTP joints and 32\% use a validated joint count. $66 \%$ use US to assess remission in RA all patients $(22 \%)$, only in those treated with biologic drugs $(28 \%)$ and in RA patients with poor 